\title{
Hijacking Rho GTPases by protein toxins and apoptosis: molecular strategies of pathogenic bacteria
}

\author{
C Fiorentini* ${ }^{\star 1}$, L Falzano ${ }^{1}$, S Travaglione ${ }^{1}$ and A Fabbri ${ }^{1}$ \\ 1 Department of Ultrastructures, Istituto Superiore di Sanità, Viale Regina Elena \\ 299 - 00161 Rome, Italy \\ * Corresponding author: C Fiorentini, Department of Ultrastructures, Istituto \\ Superiore di Sanità, Viale Regina Elena 299-00161 Rome, Italy. \\ Tel: +390649903006; Fax: +390649387140; \\ E-mail: carla.fiorentini@iss.it
}

Received 4.2.02; revised 5.8.02; accepted 23.9.02

Edited by $P$ Nicotera

\begin{abstract}
Certain bacterial toxins and type-Ill-translocated virulence factors have a peculiar property: they exert part of their actions by modulating Rho GTPases. These toxins target the actin cytoskeleton of host cells and reorganize it to their own advantage, either to facilitate macropinocytosis, which is required for invasive bacteria to enter cells, or to block pathogen sequestration by macrophages. In addition, by acting on Rho GTPases, bacteria may also interfere with the fate of host cells, favoring survival or death depending on their needs. Rho GTPases control the activation of NF- $\kappa \mathrm{B}$, which is involved in the expression of antiapoptotic proteins and mediates immunological responses as well. Here, we give a perspective on how NF- $\kappa \mathrm{B}$ may participate in linking Rhoacting toxins and apoptosis.

Cell Death and Differentiation (2003) 10, 147-152. doi:10.1038/ sj.cdd. 4401151

Keywords: Rho GTPases; bacterial protein toxins; type IIIsecreted toxins; apoptosis; NF- $\kappa \mathrm{B}$

Abbreviations: GEF, guanine exchange factor; GAP, GTPase activating protein; $\mathrm{CdA}$, Clostridium difficile toxin $\mathrm{A}$; $\mathrm{CdB}$, Clostridium difficile toxin B; LT, Letal Toxin; CNF1, cytotoxic necrotizing factor 1; CNF2, cytotoxic necrotizing factor 2; DNT, Dermonecrotizing toxin; NF- $\kappa \mathrm{B}$, nuclear factor-kB; ExoS, exoenzyme S; IL, interleukin; APC, Antigen Presenting Cell; GDI, guanine dissociation inhibitor; IAPs, inhibitors of apoptosis proteins
\end{abstract}

\section{Introduction}

Bacterial pathogens have evolved different strategies to interact with mammalian cells, including the production of protein toxins. ${ }^{1}$ Besides mediating the long-range crosstalk between bacteria and host cells, toxins serve as primary virulence factors and interact in different ways with the eukaryotic machinery, somehow favoring the survival or the spreading of bacteria. The mode of action of a large number of toxins has been unraveled in recent years. ${ }^{1}$ Certain toxins act directly on the surface of target cells either causing permeabilization of the cell membrane (pore-forming toxins) or altering normal cell signal transduction. Following endocytosis or by direct delivery, other toxins transfer an enzymatically active domain into the cytoplasm and modify cytosolic targets (Figure 1).

One recent fascinating discovery is that toxins and bacterial virulence factors frequently interfere with pathways leading to cell death (for a review see Weinrauch and Zychlinsky ${ }^{2}$ ). Poreforming toxins (Figure 1a), such as aerolysin from Aeromonas hydrophila, can induce macrophage apoptosis by allowing a massive entry of calcium into cells. ${ }^{3}$ Very recent findings also show that bacterial pore-forming toxins may translocate to host cell mitochondria and trigger apoptosis by releasing cytochrome c. ${ }^{4-6}$ Activation of T cells by superantigenic toxins (Figure 1b), such as enterotoxins from Staphylococcus aureus, may also lead to cell death by activating apoptotic pathways. $^{7}$

Rho GTPases belong to the Ras superfamily of monomeric 20-30 kDa GTP-binding proteins and, like the other Rasrelated proteins, behave as molecular switches that couple signal transduction cascades to modifications in the cell surroundings $^{8}$ (for the molecular mechanism of Rho GTPases, see Figure 2). One of the key functions of Rho GTPases is to affect the architecture of the actin cytoskeleton. ${ }^{9}$ The three main subgroups encompassing the Rho family, Rho, Rac and Cdc42, transduce extracellular signals into changes of the actin cytoskeleton, which are required for the formation of stress fibers, membrane ruffles and filopodia, respectively. Besides controlling different aspects of the actin cytoskeleton, the Rho GTPases are involved in a large variety of cellular effects, such as cell polarity, cell adhesion, phagocytosis, modulation of epithelial and endothelial cell junctions, cell migration, cell cycle progression, gene transcription, transformation and apoptosis (for a review see Symons and Settleman ${ }^{10}$ ). In fact, Rho GTPases have been implicated in both pro- and antiapoptotic signaling and in the apoptotic process itself. ${ }^{11}$

Bacteria have elaborated two molecular strategies to manipulate the Rho GTPases in host cells and interfere with apoptosis. The first consists in utilizing a protein toxin that, following entry into the cytoplasm by endocytosis, directly modifies Rho and can either activate or inhibit host cell death (Figures 1c and 3a). The second strategy involves a type III secretion system that bacteria utilize to inject effector proteins mimicking the Rho regulatory proteins GEF and GAP (Figures $1 \mathrm{~d}$ and $3 \mathrm{~b})$. By the same system, they also inject effectors that target molecules related to the apoptotic pathway. 


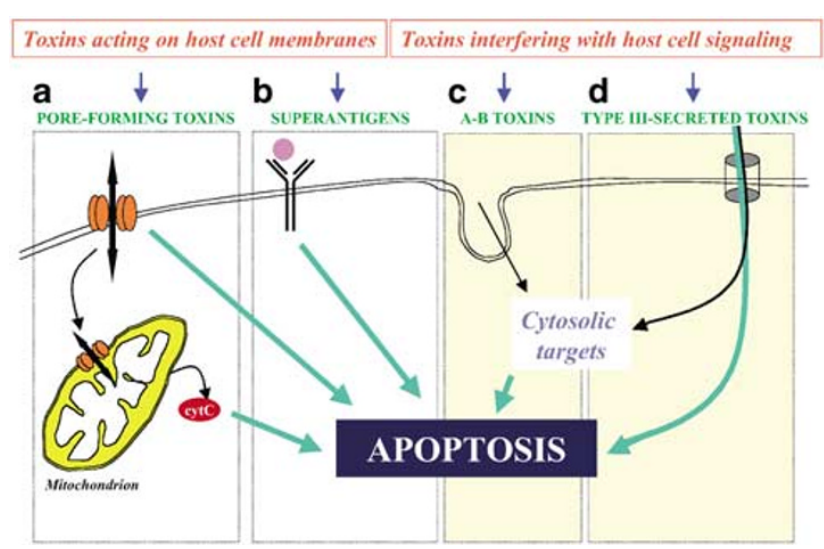

Figure 1 Bacterial toxins and apoptosis. Bacterial toxins can be divided into two main groups: toxins acting at the level of cell membrane $(\mathbf{a}, \mathbf{b})$ and toxins that must penetrate within the host cell cytosol to exert an enzymatic activity (c, d). Toxins belonging to the first group are: (a) pore-forming toxins that can insert in the lipidic core of the membrane; an example is the pore-forming $\alpha$-toxin from Staphylococcus aureus that induces apoptosis after its transfer from the plasma membrane to the outer membrane of the mitochondrion, where it provokes the release of cytochrome $c$ and the subsequent activation of caspase 3 ; and (b) superantigens that bind on cell surface receptors such as the T-cell receptor. Superantigens are able to crosslink antigen presenting cells (APCs) to the T receptor of T-lymphocytes without needing an antigenic peptide. In the absence of a costimulatory signal, however, this leads to the T-cell apoptosis. Toxins interfering with host cell signaling modify a target protein upon introduction of a catalytic moiety within the cell. (c) The so-called A-B toxins belong to this group, A being the catalytic domain and $B$ the binding domain that allows endocytosis of the molecule and the transfer of the A domain into the cytosol. A classical A-B toxin that induces apoptosis is Clostridium difficile toxin B which, by its A moiety, inactivates the Rho GTPases. Bacteria can also directly deliver virulence factors into cells (d) by locally producing an 'injecting needle' (type-III injecting system) that allows the transfer into the cytosol of a domain (comparable to the A moiety of an A-B toxin) active on a target protein. A typical archetype of type-III-secreted toxins inducing apoptosis is IpaB from Shigella spp

\section{Toxins directly modifying the Rho GTPases and apoptosis}

For the majority of pathogenic bacteria that release toxins acting directly on the GTPase molecules, the first barrier they have to circumvent is represented by an epithelium. By handling the actin cytoskeleton, the toxins they produce either favor the spreading of bacteria or allow them to survive inside a protected niche facing the host defense.

\section{Inhibitory toxins acting on switch 1 domain of Rho GTPases: commitment to apoptosis}

Bacterial toxins inactivating small GTPases most often modify a pivotal residue located in switch 1 domain (as shown in Figure 3a), which is responsible for the coupling of GTPases with their downstream effectors. The enzymatic modifications induced by Clostridium difficile toxins $\mathrm{A}$ and $\mathrm{B}(\mathrm{CdA}$ and $\mathrm{CdB}),{ }^{12} \mathrm{C}$. botulinum exoenzyme $\mathrm{C} 3^{13}$ and $C$. sordellii lethal toxin $(\mathrm{LT})^{14}$ are reported in Figure 3a. They impair the interaction of GTPases with effector molecules, thus provoking actin cytoskeleton alteration and rounding up of cells growing in a monolayer. Interestingly, depending on the strain of the producing bacteria, LT modifies Ras, Rap and Ral in addition to Rac, but not Rho. ${ }^{14}$

The Rho GTPases modified by CdA, CdB, C3 and LT are involved in the toxin-induced apoptosis via (i) disruption of the actin cytoskeleton and (ii) altered transcription of antiapoptotic genes. In fact, normal and transformed cells, both adhering and in suspension, have been reported to undergo apoptosis after toxin exposure. ${ }^{15-25}$ In some cases, this was probably because of cell rounding and detachment that followed the actin breakdown. ${ }^{15-17}$ However, changes in the actin cytoskeleton by $\mathrm{CdB}$ played an essential role in protecting cytotoxic T lymphocytes from FAS-induced apoptosis, ${ }^{26}$ this being probably because of the need of an intact cytoskeleton for the transduction of apoptotic Fas-signaling. ${ }^{27}$ Changes in the activation of transcription factors as well as altered expression of antiapoptotic genes caused by $\mathrm{CdA}$, $\mathrm{CdB}^{21}$ and $\mathrm{LT}^{28}$ may induce apoptosis with a mechanism apparently independent of perturbations of the actin cytoskeleton. Possibly, also for C3, a Rho-dependent modification of transcription of antiapoptotic genes, such as those belonging to the $\mathrm{Bcl}-2$ family, is at the basis of the apoptotic response. ${ }^{22-24}$ Moreover, since LT deactivates Ras in addition to $\mathrm{Rac} / \mathrm{Cdc} 42$, it is possible to hypothesize that this toxin may induce apoptosis also through inhibition of PI3kinase/AKT2-mediated cell survival pathway. ${ }^{29}$

It is now becoming evident that Rho GTPases do not represent the only route $\mathrm{CdA}$ and $\mathrm{CdB}$ may travel to trigger apoptosis. In fact, direct damage to mitochondria can be caused by $\mathrm{CdA}^{30}$ and a domain outside the catalytic domain might be responsible for the activation of a caspase-3dependent apoptotic response aroused by $\mathrm{CdB} .{ }^{31}$

\section{Activating toxins that act on switch 2 domain of Rho GTPases: suppression of apoptotic cell death}

The cytotoxic necrotizing factors 1 and 2 (CNF1 and CNF2) from pathogenic Escherichia coli and the dermonecrotizing toxin (DNT) from Bordetella bronchiseptica modify an amino acid that lies in switch 2 domain of Rho GTPases, blocking intrinsic and GAP-stimulated GTP hydrolysis, thereby constitutively activating the GTPases. In addition to Rho, Rac and Cdc42 are also targets for these toxins (Figure 3a) (for a review on CNF1, see Boquet and Fiorentini ${ }^{32}$ ). This leads to the reorganization of the actin cytoskeleton that influences and modifies certain functional properties of cells.

Also in the case of CNF1, the influence on apoptosis is achieved by Rho-dependent modifications of the actin cytoskeleton as well as Rho-dependent gene transcription. In fact, CNF1 protects from apoptosis induced by certain stimuli by increasing the expression of antiapoptotic genes and by preventing the mitochondrial membrane depolarization $^{33}$ as well as by augmenting the ability of cells to adhere to one another and to the extracellular matrix. ${ }^{34}$ However, CNF1 has also been reported to induce apoptosis in a uroepithelial cell line, although cytotoxic effects are probably at the basis of such a phenomenon. ${ }^{35}$

Taken altogether, the above findings suggest that toxins directly acting on Rho GTPases may influence the cell's destiny by altering the actin cytoskeleton 


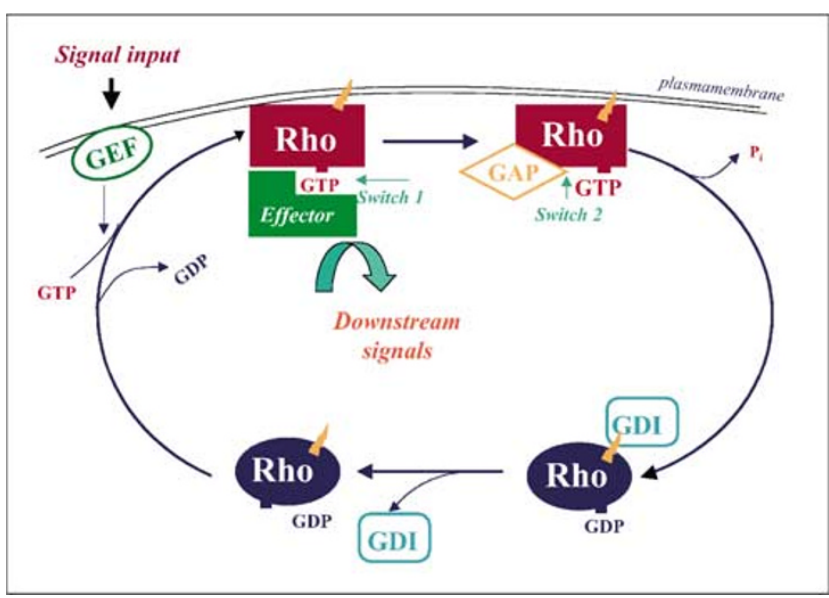

Figure 2 Rho GTPases activation/deactivation cycle. Rho GTPases are molecular switches that cycle between an inactive GDP-bound and an active GTP-bound state. Activation of Rho GTPases occurs by stimulation with a guanine exchange factor (GEF) that causes the release of GDP and the binding of GTP. In the GTP-bound form, Rho proteins undergo a conformational change in their switch 1 (sw 1) domain becoming able to interact with effector molecules and thus initiating a downstream response. Note that a major feature of Rho GTPases, as well as of other small GTP-binding proteins, is the absolute requirement for membrane attachment in order to exert their activities. In fact, small GTPases have sequences at their $\mathrm{COOH}$ termini that undergo posttranslational modification with lipid moieties (i.e. farnesyl, geranyl-geranyl, palmitoyl and methyl). This reaction allows the activated small GTPases (in the GTP-bound form) to interact with the cell membranes, where they exert their function. As soon as the activated GTPase has initiated the cellular response, a GTPase activating protein (GAP), by hydrolyzing GTP into GDP at the switch 2 (sw 2) domain, turns back these proteins to their GDP-bound state, to complete the cycle and terminate the signal transduction. Besides GEF and GAP proteins, a third factor that regulates Rho GTPases activity is the guanine dissociation inhibitor (GDI), which maintains Rho in the cytoplasm in the inactive form (linked to GDP) and, behind that, by modulating transcription. It is worth noting that Rho, Rac and $\mathrm{Cdc} 42$ are able to activate the $\mathrm{NF}-\kappa \mathrm{B}$ pathway, which plays a pivotal role in the survival/apoptosis regulation. ${ }^{36} \mathrm{~A}$ possible scheme for the connection between Rho-dependent and NF- $\kappa$ B-dependent pathways is drawn and discussed in Figure 4, in accordance with the mechanism proposed by Cammarano and Minden. ${ }^{36}$

\section{Type-III-secreted toxins mimicking the Rho GTPases and apoptosis}

Certain Gram-negative bacterial pathogens are equipped with a secretory machinery termed the type-III secretion apparatus, which is highly conserved in both animal and plant pathogens. ${ }^{37}$ Bacteria use this protein secretion pathway to deliver a set of effector proteins into the host cell cytosol, where they can usurp host cell signal transduction pathways or mimic host cell molecules. Such type-III-secreted proteins mediate a variety of events, such as macropinocytosis and bacterial invasion in epithelial cells and antiphagocytosis and apoptosis in macrophages. ${ }^{38}$ Macropinocytosis is triggered by a vast number of type-III-secreted toxins that, acting either directly on Rho GTPases or mimicking the host cell GEFs, ${ }^{39}$ favor the bacterial entry into epithelial cells (Figure $3 b$ ). However, since the promotion of this cellular activity in epithelial cells is so far not connected to apoptosis, this matter is out of the scope of this review.

The general strategy employed by these pathogenic bacteria is the block of phagocytosis and the subsequent elimination of the harmful phagocytes by apoptosis. To gain this result, a vast number of bacteria coerce the Rho GTPases
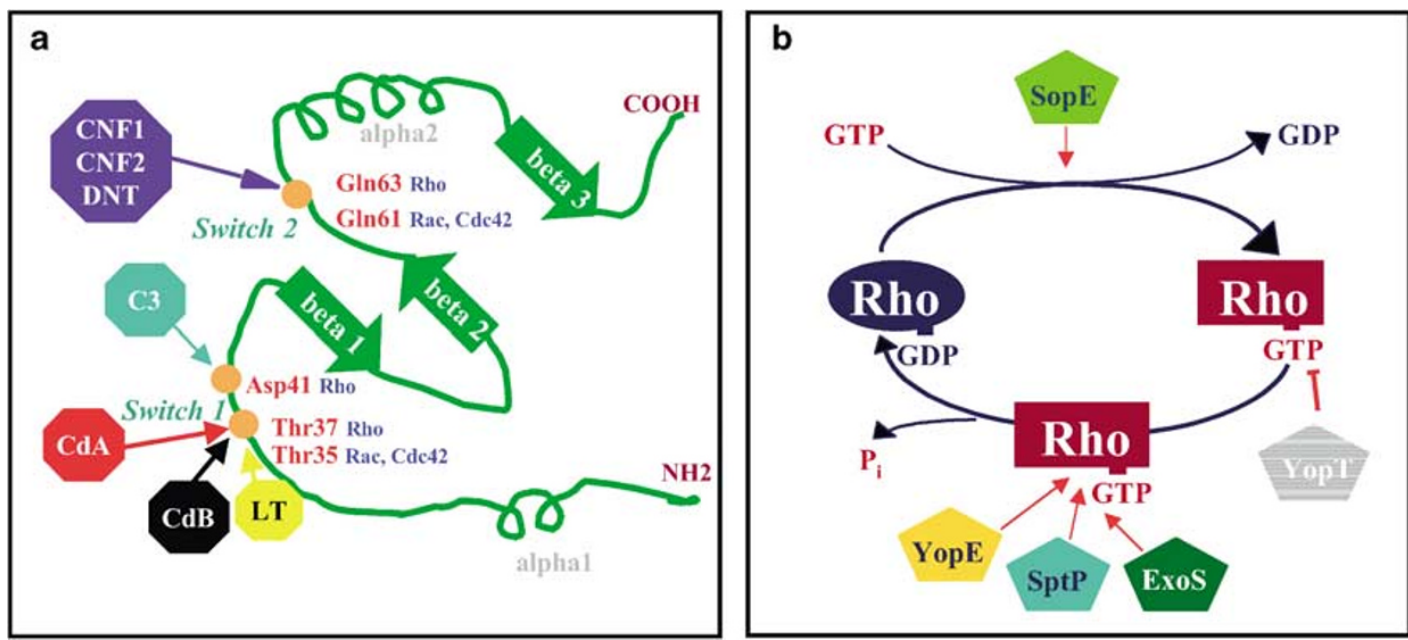

Figure 3 Hijacking of Rho GTPase by bacterial pathogens. (a) Protein toxins that directly modify Rho proteins. C3 ADP-ribosyltransferase modifies Rho at Asn41, an amino acid located in switch 1 domain. Although ADP-ribosylated Rho can still bind to the effectors, it has a greater affinity for Rho-GDI than the nonmodified form. It therefore remains associated to Rho-GDI and it is not recruited to the membrane, thereby losing its activity. CdA, CdB and LT from C. sordellii glucosylate Rho GTPases at Thr35/Thr37 in switch 1 domain, thus impairing the interaction of Rho GTPases with the effector molecules. CNF1, CNF2 and DNT deamidate the Rho GTPases at Gln63/Gln61 that are located in switch 2 domain. This modification inhibits the intrinsic and GAP-stimulated GTP hydrolyzing activity of Rho proteins, turning on constitutively the GTPases. (b) Type-III-secreted toxins mimicking host cell regulatory proteins that signal Rho GTPases. Certain invasive bacteria such as Salmonella, Yersinia or Shigella activate the Rho GTPases to trigger their own phagocytosis in nonprofessional phagocytes or to inhibit macrophage phagocytosis. Their strategy consists in the introduction of an effector molecule directly into the cytosol of the host cells by a type-lll-secretion mechanism. SopE from Salmonella typhimurium is an exchange factor for Cdc42 and Rac. YopE from Yersinia, SptP from Salmonella and ExoS from P. aeruginosa play a role as GAP proteins. In contrast to the other typeIII-secreted toxins, YopT cleaves directly Rho-GTP on its carboxy-terminus removing the geranyl-geranyl post-translational modification and thereby inducing Rho detachment from the cell membrane 
that are central to the organization of the actin cytoskeleton (a scheme on the mechanisms employed is shown in Figure 5). Exoenzyme $S$ (ExoS) plays an, as yet, undefined role in the pathogenesis of the opportunistic pathogen Pseudomonas aeruginosa. $^{40}$ It is a bifunctional protein, with two distinct domains, both able to mediate changes in the cytoskeleton. The C-terminus moiety of ExoS ADP-ribosylates Ras at Arg41 and Arg-128 residues, thus inactivating Ras and blocking Ras-dependent signaling pathways. The $\mathrm{N}$-terminus domain exhibits an 'arginine finger' sequence that acts as a GAP for Rac and Cdc42. ${ }^{40}$ The ExoS N-terminus has a limited amino acid homology to YopE from Yersinia pseudotubercolosis that also acts as a GAP stimulating the GTPase activity of Cdc42 and Rac but not of other small GTPases. ${ }^{41}$ Yersinia YopT is a cystein metalloprotease that cleaves the carboxy terminus end of Rho GTPases, causing their release from the plasma membrane and the consequent disruption of the actin cytoskeleton. ${ }^{42}$ In all cases, the inactivation of Rho interrupts signaling events that are necessary for the actin cytoskeleton reshape, thus potentially contributing to antiphagocytosis.

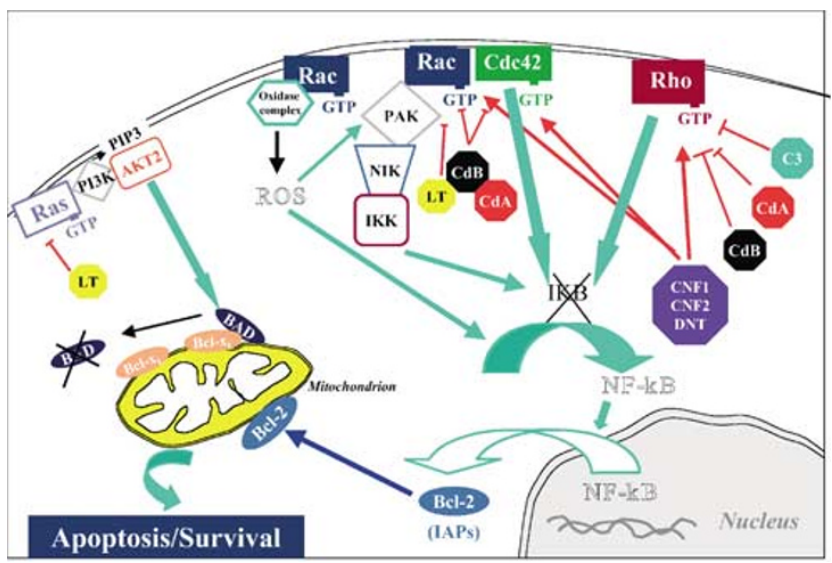

Figure 4 Apoptosis by toxins directly modifying the Rho GTPases. As already shown in Figure 3a, certain protein toxins act directly on Rho GTPases to either activate (CNF1, CNF2, DNT) or inhibit (CdB, CdA, C3, LT) them. The scheme exemplifies, in accordance with the current literature, our view on how Rho GTPase activity and apoptosis may be connected and point out NF- $\kappa$ B as a cardinal player of the game. Once stimulated, all the three subfamilies Rho, Rac and $\mathrm{Cdc} 42$ are able to, in turn, activate the NF- $\kappa \mathrm{B}$ pathway although by different modalities. Rac (activated by the GEF Dbl) first activates PAK, which activates $\mathrm{NIK}$, which in turn activates IKK, thus phosphorylating IKB. IKB is subsequently degraded in the proteasome thereby freeing NF- $\kappa \mathrm{B}$, which then enters the nucleus, binds to DNA and activates transcription. Activation of IKK may be increased by Rac-dependent generation of ROS that, besides directly activating $\mathrm{NF}-\kappa \mathrm{B}$, also stimulates PAK. By contrast, both Rho- and Cdc42-dependent activation of NF- $\kappa$ B by phosphorylation of IKB are not due to IKK but to so far unknown kinases. The main role of $\mathrm{NF}-\kappa \mathrm{B}$ in the survival/apoptosis regulation is the production of antiapoptotic factors such as proteins belonging to the Bcl-2 family or inhibitors of apoptosis proteins (IAPs). Bcl-2 localizes on the mitochondrial surface, impairing the release of the proapoptotic cytochrome $c$ and thus favoring survival. The block of the NF- $\kappa$ B pathway by inhibition of Rho GTPases commits cells to apoptosis. Besides inhibiting Rac, LT also acts on Ras thus manipulating a parallel and distinct pathway that control apoptosis. Activated Ras activates the PI3-kinase, which in turn activates AKT2. This last kinase phosphorylates the proapoptotic protein BAD causing its degradation into the proteasome, thus reducing the binding of BAD to the antiapoptotic $B c l-X_{L}$ and hence favoring survival. Such a Ras-dependent pathway is inhibited by LT, a toxin that may trigger apoptosis via this alternative pathway, in analogy to what occurs with Rac inhibition

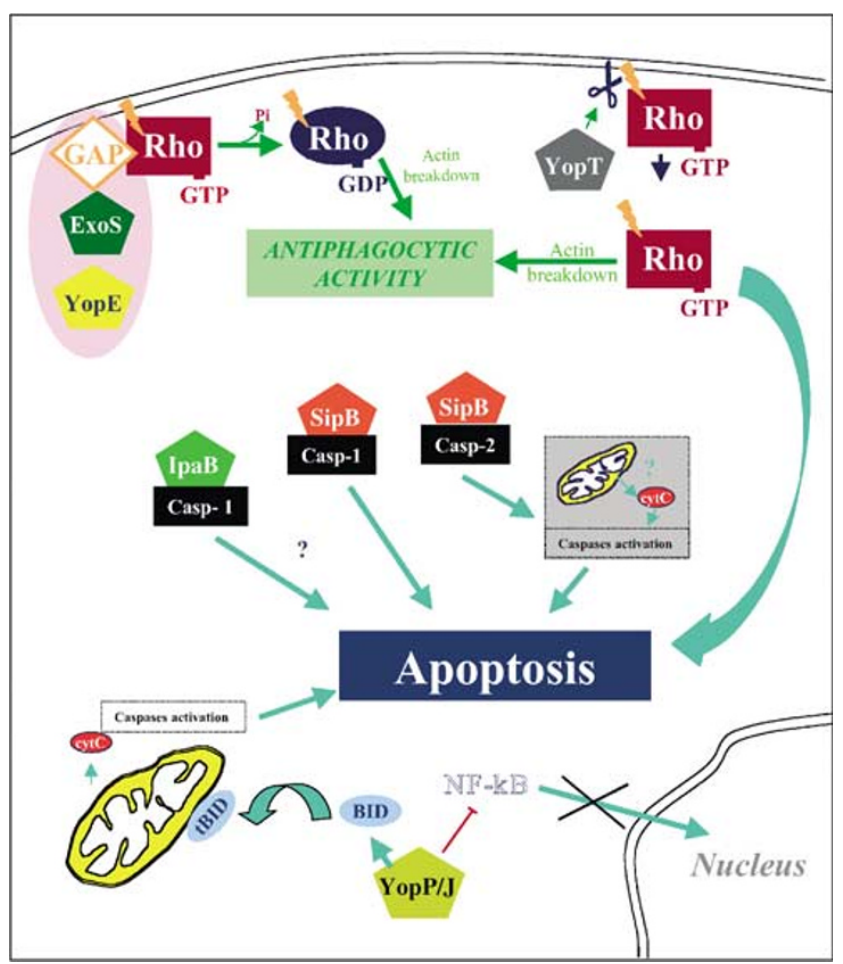

Figure 5 Type-III-secreted toxins: molecular strategies to foil macrophages. Several bacterial pathogens utilize type-III-secreted effector molecules that usurp the control of Rho GTPases to exert an antiphagocytic activity. YopE and ExoS, which mimic the host cell GAP, interact with Rho-GTP, to induce GTP hydrolysis and the release of Rho-GDP from the plasma membrane. YopT cleaves directly Rho-GTP causing its detachment from the plasma membrane. In both cases, the consequent Rho inactivation interrupts signaling events necessary for the actin cytoskeleton reorganization and thus potentially contributes to antiphagocytosis. Besides impairing their own capture by macrophages, certain bacteria also activate the apoptotic pathway by type-III-secreted toxins. YopT, besides inducing the actin cytoskeleton breakdown, commits cells to apoptosis by directly modifying the Rho GTPases. IpaB and SipB can bind to and presumably activate caspase-1. How caspase-1 can trigger apoptosis is still under debate. In addition to caspase-1, SipB directly binds to caspase-2 switching on an apoptotic pathway that also involves the activation of caspase-3, -6, -8 and the release of cytochrome $\mathrm{c}$ from mitochondria. YopP/J also induces macrophage apoptosis probably by preventing the nuclear translocation of the antiapoptotic factor NF$\kappa \mathrm{B}$. YopP/J also generates a truncated form of the proapoptotic protein BID (tBID) that, upon translocation to mitochondria, induces the release of cytochrome $c$, activation of executioner caspases and apoptosis

The first bacterium shown to induce apoptosis was Shigella flexneri, which specifically kills cultured macrophages but not epithelial cells. ${ }^{43}$ Macrophages undergo apoptosis and release large quantities of the proinflammatory cytokine interleukin (IL)-1. The IpaB protein is required for such effects, independent of its role in cell entry. Inside cells, IpaB binds to caspase-1, a protease that cleaves pro-IL-1 to release the mature protein and, presumably, such a binding also activates apoptosis. ${ }^{44}$ Salmonella spp. also induce phagocyte apoptosis by using a type-III-secreted protein, SipB, which directly activates the host cell's apoptotic and inflammatory pathways by targeting caspase- $1,{ }^{45}$ in the same vein of IpaB. More recent evidence, however, shows that SipB activates the apoptotic machinery by also regulating caspase-2 as well as caspase- $3,-6,-8$ and the release of cytochrome $c$ from mitochondria. ${ }^{46}$ Mitochondria, however, are untouched in 
Salmonella-infected macrophages. It is possible that cytochrome $c$ feeds back on the caspase cascade and accelerates the apoptotic process.

Within the host cell, type-III-secreted toxins from Yersinia act to inhibit phagocytosis and induce apoptosis. ${ }^{47}$ The plant virulence factor AvrPphB (which does not target the Rho GTPases) acts as YopT, modifying a target molecule that blocks the infection by triggering apoptosis of the plant defense system (hypersensitive response). ${ }^{48}$ In general, modifications of target molecules by plant virulence factors are recognized by a detection system of the host ( $R$ factor), which responds by inducing cell death. As stated by Schneider, ${ }^{48}$ the pathogen may double-cross the host and turn that to its own advantage. This could be also true for bacterial virulence factors targeting mammalian cells. For instance, YopP/J from Yersinia plays an anti-inflammatory role beneficial to the bacteria by preventing the activation of $\mathrm{NF}-\kappa \mathrm{B}$, but also induces apoptosis because NF- $\kappa \mathrm{B}$ is required for cell survival. ${ }^{49} \mathrm{YopP} / \mathrm{J}$ also generates a truncated form of the proapoptotic protein BID (tBID) that, upon translocation to mitochondria, induces the release of cytochrome $c$, the activation of executioner caspases and apoptosis. ${ }^{50}$ Therefore, modification of Rho GTPases themselves, or their downstream effects (e.g. actin cycloskeleton changes), might be detected by the host cell, which will hence induce apoptosis.

\section{Orchestration of host cell pathways by Rho-acting toxins: apoptosis at the service of bacterial pathogens?}

Bacterial toxins are long-range acting virulence factors whose main goal is to allow the survival and the spreading of pathogenic microbes. They may achieve this by helping bacteria to reach a protected niche wherein they can survive and multiply, or by impairing mechanisms of the immune system thus weakening the host defense. Rho, Rac and Cdc42 GTPases have been selected during evolution as targets for many toxins. Their selection is probably because of the pivotal role they play in the hierarchy of regulatory molecules that coordinate the assembly of the actin cytoskeleton, which is the main 'engine' of cellular functions such as locomotion, cell adhesion and phagocytosis. Indeed, the actin cytoskeleton is linked to nearly all the essential processes driving cell development and differentiation. Conceivably, modifications induced by toxins to Rho GTPases may consequently result in life or death of their target cells.

Most likely, bacteria elaborate such toxins for the main purpose of modifying the actin network and probably not to interfere with the apoptotic pathway. CdA, CdB or LT toxins disrupt permanently the actin cytoskeleton, possibly to alter digestive tract functionality. The consequent diarrhea allows the spreading of bacteria outside the host, thus facilitating their dispersion to another host, in a system similar to that employed by cholera toxin. In the case of CNF1 or DNT, activation of Rho GTPases is probably accomplished to allow bacteria to invade epithelial cells that also become resistant to apoptosis and thus provide a safe spot inaccessible to the host defenses. Mimicry of Rho regulatory proteins GEF and
GAP by Salmonella type-III-secreted toxins (SopE and SptP) results in prophagocytic activity that also requires the manipulation of the actin cytoskeleton. These two activities must be exactly balanced, otherwise an excessive stimulation or inhibition of the Rho GTPases will modify the host cell fate. Also, blocking phagocytosis by type-III-translocated toxins (YopT, YopE, ExoS) avoids the ruin of bacteria that become offensive and may exploit apoptosis to foil the macrophage.

By modifying Rho GTPases, however, bacteria certainly perturb other pathways of regulation, one of the most important being the regulation of NF- $\kappa \mathrm{B} .{ }^{51}$ Handling the NF$\kappa \mathrm{B}$ pathway is a double-edged sword for bacteria since, besides controlling the expression of antiapoptotic proteins that affect the susceptibility of cells to apoptosis, NF- $\kappa$ B is also the principal transcription factor for the response against pathogens (both the innate and adaptative immune system). ${ }^{52}$ Therefore, it is not clear whether NF- $\kappa$ B activation in response to Rho-acting toxins benefits the host or the bacterial pathogen. A question arises: are bacterial pathogens really masters of their own destiny?

\section{Acknowledgments}

We are grateful to $\mathrm{W}$. Malorni for a critical reading of the manuscript.

\section{References}

1. Schiavo $G$ and van der Goot FG (2001) The bacterial toxin toolkit. Nature 2: 530-537

2. Weinrauch $Y$ and Zychlinsky $A$ (1999) The induction of apoptosis by bacterial pathogens. Annu. Rev. Microbiol. 53: 155-187

3. Nelson KL, Brodsky RA and Buckley JT (1999) Channels formed by subnanomolar concentrations of the toxin aerolysin trigger apoptosis of $T$ lymphomas. Cell Microbiol. 1: 69-74

4. Muller A, Rassow J, Grimm J, Machuy N, Meyer TF and Rudel T (2002) VDAC and the bacterial porin PorB of Neisseria gonorrhoeae share mitochondrial import pathways. EMBO J. 21: 1916-1929

5. Bantel H, Sinha B, Domschke W, Peters G, Sculze-Osthoff $K$ and Janicke RU (2001) Alpha-toxin is a mediator of Staphylococcus aureus-induced cell death and activates caspases via the intrinsic death pathway independently of death receptor signalling. J Cell Biol. 155: 637-648

6. Galmiche A, Rassow J, Doye A, Cagnol S, Chambard JC, Contamin S, de Thillot V, Just I, Ricci V, Solcia E, Van Obberghen E and Boquet P (2000) The $\mathrm{N}$-terminal $34 \mathrm{kDa}$ fragment of Helicobacter pylori vacuolating cytotoxin targets mitochondria and induces cytochrome c release. EMBO J. 19: 6361-6370

7. Lavoie PM, McGrath H, Shoukry NH, Cazenave PA, Sekaly RP, Thibodeau J (2001) Quantitative relationship between MHC class II-superantigen complexes and the balance of T cell activation versus death. J. Immunol. 166:7229-7237

8. Takai Y, Sasaki T and Matozaki T (2001) Small GTP-binding proteins. Physiol. Rev. 81: 153-208

9. Hall A (1998) Small GTPases and the actin cytoskeleton. Science 279: 509-514

10. Symons $M$ and Settleman $J(2000)$ Rho family GTPases: more than simple switches. Trends Cell Biol. 10: 415-419

11. Coleman ML and Olson MF (2002) Rho GTPase signalling pathways in the morphological changes associated with apoptosis. Cell Death Differ. 9: 493-504

12. Just I, Hofman F and Aktories K (2000) Molecular mechanisms of action of the large Clostridial cytotoxins. In Bacterial Proteins Toxins (Handbook of Experimental Pharmacology, vol. 145), Aktories K and Just I, eds (Berlin: Springer) pp. 307-327 
13. Aktories K, Barth, $\mathrm{H}$ and Just I (2000) Clostridium botulinum C3 exoenzyme and C3-like transferases. In Bacterial Proteins Toxins (Handbook of Experimental Pharmacology, vol. , 145). Aktories K and Just I, eds (Berlin: Springer) pp. 207-225

14. Popoff MR, Chaves-Olarte E, Lemichez E, von Eichel-Streiber C, Thelestam M, Chardin P, Cussac D, Antonny B, Chavrier P, Flatau G, Giry M, de Gunzburg J and Boquet $P$ (1996) Ras, Rap and Rac small GTP-binding proteins are targets for Clostridium sordellii lethal toxin glucosylation. J. Biol. Chem. 271: 1021710224

15. Fiorentini C, Donelli G, Nicotera P and Thelestam M (1993) Clostridium difficile toxin $\mathrm{A}$ elicits $\mathrm{Ca}^{2+}$-independent cytotoxic effects in cultured normal rat intestinal cript cells. Infect. Immun. 61: 3988-3993

16. Mahida YR, Makh S, Hyde S, Gray T and Borriello SP (1996) Effect of Clostridium difficile toxin A on human intestinal epithelial cells: induction of interleukin 8 production and apoptosis after cell detachment. Gut 38: 337-347

17. Mahida YR, Galvin A, Makh S, Hyde S, Sanfilippo L, Borriello SP and Sewell HF (1998) Effect of Clostridium difficile toxin A on human colonic lamina propria cells: early loss of macrophages followed by T-cell apoptosis. Infect. Immun. 66: $5462-5469$

18. Fiorentini C, Fabbri A, Falzano L, Fattorossi A, Matarrese P, Rivabene R and Donelli G (1998a) Clostridium difficile toxin B induces apoptosis in intestinal cultured cells. Infect. Immun. 66: 2660-2665

19. Le Gall M, Chambard JC, Breittmayer JP, Grall D, Pouyssegur J, and Van Obberghen-Schilling E (2000) The p42/p44 MAP kinase pathway prevents apoptosis induced by anchorage and serum removal. Mol. Biol. Cell 11: 11031112

20. Anderson RJ, Ray CJ and Popoff MR (2000) Evidence for rho protein regulation of renal tubular epithelial cell function. Kidney Int. 58: 1996-2006

21. Gomez J, Martinez C, Giry M, Garcia A and Rebollo A (1997) Rho prevents apoptosis through Bcl-2 expression: implications for interleukin-2 receptor signal transduction. Eur. J. Immunol. 27: 2793-2799

22. Moorman JP, Bobak DA and Hahn CS (1996) Inactivation of the small GTP binding protein Rho induces multinucleate cell formation and apoptosis in murine T lymphoma EL4. J. Immunol. 156: 4146-4153

23. Bobak D, Moorman J, Guanzon A, Gilmer L and Hahn C (1997) Inactivation of the small GTPase Rho disrupts cellular attachment and induces adhesion-dependent and adhesion-independent apoptosis. Oncogene 15: 2179-2189

24. Henning SW, Galandrini R, Hall A and Cantrell DA (1997) The GTPase Rho has a critical regulatory role in thymus development. EMBO J. 16 2397-2407

25. Calderon GM, Torres-Lopez J, Lin TJ, Chavez B, Hernandez M, Munoz O, Befus AD and Enciso AJ (1998) Effects of toxin A from Clostridium difficile on mast cell activation and survival. Infect. Immun. 66: 2755-2761

26. Subauste MC, Von Herrath M, Benard V, Chamberlain CE, Chuang TH, Chu K, Bokoch GM and Hahn KM (2000) Rho family proteins modulate rapid apoptosis induced by cytotoxic T lymphocytes and Fas. J. Biol. Chem. 275: 9725-9733

27. Parlato S, Giammarioli AM, Logozzi M, Lozupone F, Matarrese $P$, Luciani $F$, Falchi M, Malorni W and Fais S (2000) CD95 (APO-1/Fas) linkage to the actin cytoskeleton through ezrin in human $\mathrm{T}$ lymphocytes: a novel regulatory mechanism of the CD95 apoptotic pathway. EMBO J. 19: 5123-5134

28. Linseman DA, Laessing T, Meintzer MK, McClure M, Barth $\mathrm{H}$, Aktories $\mathrm{K}$ and Heindenreich (2001) An essential role for Rac/Cdc42 GTPases in cerebellar granule neuron survival. J. Biol. Chem. 276: 39123-39131

29. Jiang K, Coppola D, Crespo NC, Nicosia SV, Hamilton AD, Sebti SM and Cheng JQ (2000) The phosphoinositide 3-OH kinase/AKT2 pathway as a critical target for farnesyltransferase inhibitor-induced apoptosis. Mol. Cell Biol. 20: $139-148$

30. He D, Hagen SJ, Pothoulakis C, Chen M, Medina ND, Warny M and LaMont JT (2000) Clostridium difficile toxin A causes early damage to mitochondria in cultured cells. Gastroenterology 119: 139-150

31. Qa Dan M, Ramsey M, Daniel J, Spyres LM, Safiejko-Mroczka B, Ortiz-Leduc W and Ballard JD (2002) Clostridium difficile toxin B activates dual caspasedependent and caspase-independent apoptosis in intoxicated cells. Cell. Microbiol. 4: 425-434
32. Boquet $P$ and Fiorentini $C$ (2000) The cytotoxic necrotizing factor 1 from Escherichia coli. In Bacterial Proteins Toxins (Handbook of Experimental Pharmacology, vol. , 145) Aktories K. and Just I, eds (Berlin: Springer) pp. 361-379

33. Fiorentini C, Matarrese $P$, Straface $E$, Falzano L, Fabbri A, Donelli $G$ Cossarizza A, Boquet P and Malorni W (1998) Toxin-induced activation of Rho GTP-binding protein increases $\mathrm{Bcl}-2$ expression and influences mitochondrial homeostasis. Exp. Cell. Res. 242: 341-350

34. Fiorentini C, Matarrese P, Straface E, Falzano L, Donelli G, Boquet $P$ and Malorni W (1998) Rho-dependent cell spreading activated by E. coli cytotoxic necrotizing factor 1 hinders apoptosis in epithelial cells. Cell Death Differ. 5 : 921-929

35. Mills M, Meysick KC and O'Brien AD (2000) Cytotoxic necrotizing factor type 1 of uropathogenic Escherichia coli kills cultured human uroepithelial 5637 cells by an apoptotic mechanism. Infect. Immun. 68 : 5869-5880

36. Cammarano MS and Minden A (2001) Dbl and the Rho GTPases activate NFkB by IkB kinase (IKK)-dependent and IKK-independent pathways. J. Biol. Chem. 276: 25876-25882

37. Cornelis GR and Van Gijsegem F (2000) Assembly and function of type III secretory systems. Ann. Rev. Microbiol. 54: 735-774

38. Knodler LA, Celli J and Finlay BB (2001) Pathogenic trickery: deception of host cell processes. Nature 2: $578-588$

39. Steele-Mortimer O, Knodler LA and Finlay BB (2000) Poisons, ruffles and rockets: bacterial pathogens and the host cell cytoskeleton. Traffic 1: 107-118

40. Goehring UM, Schmidt G, Pederson KJ, Aktories K and Barbieri JT (1999) The $\mathrm{N}$-terminal domain of Pseudomonas aeruginosa exoenzyme $\mathrm{S}$ is a GTPaseactivating protein for Rho GTPases. J. Biol. Chem. 274: 36 369-36 372

41. Von Pawel-Rammingen U, Telepnev MV, Schmidt G, Aktories K, Wolf-Watz H and Rosqvist R (2000) GAP activity of the Yersinia YopE cytotoxin specifically targets the Rho pathway: a mechanism for disruption of actin microfilament structure. Mol. Microbiol. 36: 737-748

42. Shao F, Merritt PM, Bao Z, Innes RW and Dixon JE (2002) A Yersinia effector and a Pseudomonas avirulence protein define a family of cysteine proteases functioning in bacterial pathogenesis. Cell 109: $575-588$

43. Zychlinsky A, Prevost MC, Holland IB and Sansonetti PJ (1992) Shigella flexneri induces apoptosis in infected macrophages. Nature 358: 167-169

44. Zychlinsky A, Kenny B, Menard R, Prevost MC, Holland IB and Sansonetti PJ (1994) IpaB mediates macrophage apoptosis induced by Shigella flexneri. Mol. Microbiol. 11: 619-627

45. Hersh D, Monack DM, Smith MR, Ghori N, Falkow S and Zychlinsky A (1999) The Salmonella invasin SipB induces macrophage apotosis by binding to caspase-1. Proc Natl. Acad. Sci. 96: 2396-2401

46. Jesenberger V, Procyk KJ, Yuan J, Reipert S and Baccarini M (2000) Salmonella-induced caspase-2 activation in macrophages: a novel mechanism in pathogen-mediated apoptosis. J. Exp. Med. 192: 1035-1045

47. Cornelis GR (2000) Molecular and cell biology aspects of plague. Proc. Natl. Acad. Sci. 97: 8778-8783

48. Schneider DS (2002) Plant immunity and film noir: what gumshoe detectives can teach us about plant-pathogen interactions. Cell 109: $537-540$

49. Ruckdeschel K, Harb S, Roggeznkamp A, Hornef M, Zumbihl R, Kohler S, Heesemann J and Rout B (1998) Yersinia enterocolitica impairs activation of transcription factor NF-kappaB: involvement in the induction of programmed cell death and the suppression of the macrophage tumor necrosis factor alpha production. J. Exp. Med. 187: 1069-1079

50. Denecher G, Declercq W, Geuijen CA, Boland A, Benabdillah R, van Gurp M, Sory MP, Vandenabeele P and Cornelis GR (2001) Yersinia enterocolitica YopP-induced apoptosis of macrophages involves the apoptotic signaling cascade upstream of bid. J. Biol. Chem. 276: 19706-19714

51. Perona R, Montaner S, Saniger L, Sanchez-Perez I, Bravo R and Lacal JC (1997) Activation of the nuclear factor-kappaB by Rho, CDC42, and Rac-1 proteins. Genes Dev. 11:463-475

52. Tato CM and Hunter CA (2002) Host-pathogen interactions: subversion and utilization of the NF- $\kappa$ B pathway during infection. Infect. Immun. 70: 33113317 